\title{
Remote Measurement of Cognitive Stress via Heart Rate Variability
}

\author{
Daniel McDuff ${ }^{1}$, Sarah Gontarek ${ }^{2}$ and Rosalind Picard ${ }^{1}$
}

\begin{abstract}
Remote detection of cognitive load has many powerful applications, such as measuring stress in the workplace. Cognitive tasks have an impact on breathing and heart rate variability (HRV). We show that changes in physiological parameters during cognitive stress can be captured remotely (at a distance of $3 \mathrm{~m})$ using a digital camera. A study $(n=10)$ was conducted with participants at rest and under cognitive stress. A novel five band digital camera was used to capture videos of the face of the participant. Significantly higher normalized low frequency HRV components and breathing rates were measured in the stress condition when compared to the rest condition. Heart rates were not significantly different between the two conditions. We built a person-independent classifier to predict cognitive stress based on the remotely detected physiological parameters (heart rate, breathing rate and heart rate variability). The accuracy of the model was $85 \%(35 \%$ greater than chance).
\end{abstract}

\section{INTRODUCTION}

Heart rate variability (HRV) is a commonly used measure of autonomic nervous system (ANS) activity. The two branches of the ANS are the sympathetic nervous system (SNS) and parasympathetic nervous system (PNS) which dynamically control the beat-to-beat differences of the heart. The HRV low frequency (LF) component is modulated by baroreflex activity and contains both sympathetic and parasympathetic activity [1]. The high frequency (HF) component reflects parasympathetic influence on the heart, it is connected to respiratory sinus arrhythmia (RSA). An estimate of sympathetic modulation (the sympatho/vagal balance) can be made by considering the LF/HF power ratio.

Cognitive tasks (such as mental arithmetic) have an impact on HRV [2], [3], [4]. Those under mental stress showed reduced HF HRV components compared to a control group [3]. During an attention task lower total HRV power was observed, in addition to a change in heart rate, when compared to baseline [4]. It may be possible to use spectral analysis of HRV to predict such things as the optimal work time under mental stress [5]. Automatic detection of cognitive load has much potential in such a context. Although nonverbal cues such as facial expressions can be useful in detecting cognitive states (such as concentration) [6], in many cases people do not exhibit facial muscle movements. Thermal imaging has been used to remotely capture stress [7] however the cost of the cameras is high. Remote measurement of cognitive stress

\footnotetext{
*This work was supported by the MIT Media Lab consortium.

1 Daniel McDuff and Rosalind Picard are with the Media Laboratory, Massachusetts Institute of Technology, Cambridge, MA 02139 USA. \{djmcduff, picard $\}$ at media.mit.edu

2 Sarah Gontarek is with the Massachusetts Institute of Technology, Cambridge, MA 02139 USA. sgontarek at mit.edu
}

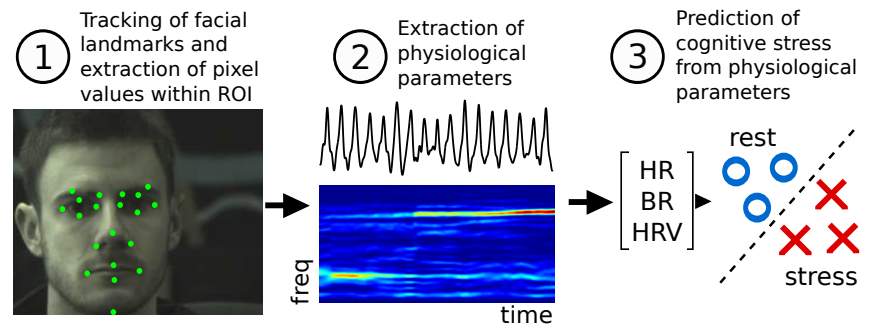

Fig. 1. Overview of the automated system for prediction of cognitive stress from remotely measured physiology. 1) Facial landmarks detected and color channel information extracted from the ROI, 2) BVP extracted from color channel signals and HR, BR and HRV parameters calculated, 3) physiological features used to predict restful state or cognitive stress state.

via HRV captured from digital cameras could allow for lowcost non-contact measurement of attention, concentration or engagement and be useful in the workplace and education.

The gold standard for HRV measurement is the electrocardiogram (ECG). However, high degrees of agreement (in both the temporal and frequency domains) have been observed between measurements of HRV derived from ECG and measurements made using photoplethysmography (PPG) [8]. PPG is a low-cost and non-invasive technique for measuring the cardiovascular blood volume pulse (BVP) through variations in transmitted or reflected light [9].

Recently, it has been shown that it is possible to recover the blood volume pulse (BVP) from images of the human face [10]. Furthermore, it is possible to do so without a dedicated light source and with a low-cost digital camera [11], [12]. Small changes to the color band sensitivities of a standard digital camera can also improve the accuracy of the BVP recovered from video images and allow equivalent measurements to be made from greater distances [13]. High correlations between contact sensor measurements and these remote measures of heart rate, breathing rate and HRV low and high frequency components have been shown. Balakrishnan et al. [14] presented a method for recovering the BVP waveform from motion of the human head allowing the heart rate to be detected even if no skin is visible. However, their system was out performed by camera PPG measurements in some cases and is susceptible to rigid head motions.

This paper addresses remote measurement of subtle changes in HR, BR and HRV and classification of restful and stress states based on these features. In the remainder of this paper we will: 1) present an experiment and results that show measurable changes in physiology using a digital camera, 2) demonstrate that these can allow remote capture of changes between a restful state and under cognitive stress, 3 ) build and test a classifier for predicting a restful state and 


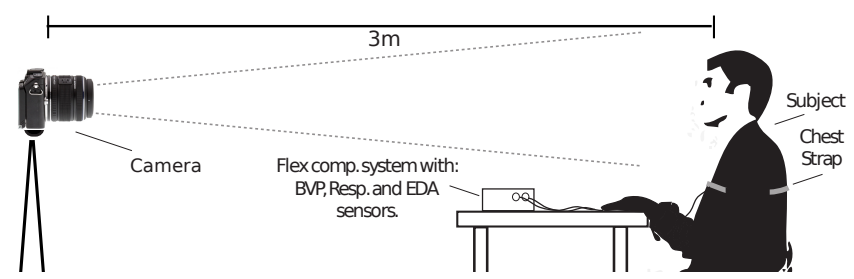

Fig. 2. Experimental set-up. Contact measurements of the blood volume pulse and electrodermal activity were collected using finger sensors and breathing measured using a chest strap. A camera, placed $3 \mathrm{~m}$ from the participant, was used to capture videos images at 30fps, 960x720 resolution.

one of cognitive stress based on physiological features.

\section{DATA COLLECTION}

\section{A. Set-Up}

Camera: The camera used to collect the video sequences was a digital single-lens reflex (DSLR) camera with a standard Zuiko 50mm lens. The camera's sensor has the capability of capturing five color bands which includes the typical three color band sensors (red, green and blue (RGB)) and also cyan and orange frequency band sensors (RGBCO). Previous work suggested that the combination of the cyan, orange and green bands was the best for recovering physiological parameters [13] and considerably outperforms the RGB signal combination, this was true across a number of participants. The sensitivity profile of the camera can be found in [13].

We use an automated method for recovering the heart rate, breathing rate and HRV spectrograms from the recorded videos presented in [13]. Figure 3 shows an overview of the approach. The videos were exported in an uncompressed format. The physiological and video recordings were analyzed offline using custom software written in MATLAB (The Mathworks, Inc.). The facial region of interest was segmented and color channel signals extracted by taking a spacial average of the pixel values in each frame. Independent Component Analysis (ICA) was used to recover source signals from the color channel signals and the signal with the strongest BVP signal selected. BVP peaks were detected and used to calculate the HRV spectrogram. We construct the spectrograms by calculating the power spectral density (PSD) from the IBIs for a moving window over the whole session. The Lomb periodogram was used to obtain the PSD over a 60 second moving window. The sessions were two minutes in length, the step size was one second.

Contact Measurements: We collected contact PPG and respiration measurements to validate the remote physiological measurements. PPG, respiration and electrodermal activity were recorded using FDA-approved sensors (Flexcomp Infiniti by Thought Technologies, Inc.). PPG and electrodermal activity (EDA) were measured with finger sensors and respiration with a chest strap. The EDA was measured from the middle and ring fingers of both hands and the BVP from the left index finger.

To calculate the HRV spectrogram using the PPG measurements from the contact finger sensor, peak detection was performed on the PPG waveform with a moving time window of length 0.25 seconds. The Lomb periodogram was again used to obtain the PSD over a 60 second moving window.

\section{B. Experiments}

This study was approved by the Institutional Review Board of the Massachusetts Institute of Technology. All experiments were conducted indoors and with a varying amount of sunlight and indoor illumination. Participants were seated and the data were recorded on a laptop (Toshiba, Windows 7). Our experiments featured 10 healthy participants of both genders (seven females), different ages (18-30) and skin color. During the experiment participants were seated approximately $3 \mathrm{~m}$ away from the camera and asked to face the camera while the videos were recorded. Figure 2 shows the set-up used to record the data. Two minute recordings of the participants were taken, the contact measurements and video sequences were synchronized by simultaneously starting both recordings.

Measurements at rest: In the first experiment participants were asked to sit still, look toward the camera and relax. The video and physiological recordings were captured for two minutes. For one of the participants in the rest condition the contact finger BVP measurements were noisy due to motion artifacts, this session was not used for the validation of the remote measurements.

Measurements under stress: In the second experiment participants were asked to perform a mental arithmetic task (MAT) silently (without talking). Starting with the number 4000 they were required to subtract 7 , then subtract 7 again, and so on, as quickly as possible. The video and physiological recordings were captured for two minutes. The participants started the task immediately after the recordings were started. In order to increase the cognitive stress induced we told the participants that they were competing against the other people to reach the lowest number. All the participants reported experiencing more stress in this condition.

\section{Quantification of Physiological Parameters}

For both the camera and contact measurements we calculated the HR, BR and HRV low frequency (LF) and high frequency (HF) components. HR was calculated as $60 / \overline{I B I}$, where $\overline{I B I}$ is the mean of the inter-beat intervals. $\mathrm{BR}$ can be estimated from the dominant high frequency component of the HRV [16]. We determine the BR from the center frequency of the highest peak $\left(f_{\text {HFpeak }}\right)$ between 0.15 and $0.4 \mathrm{~Hz}$ of the HRV power spectrum. For the contact measurements the BR was calculated as the frequency of the dominant peak $f_{\text {resp. }}$ in the PSD of the recorded respiratory waveform as $60 * f_{\text {resp. }}$. The LF and HF powers of the HRV were calculated as the area under the PSD curve corresponding to $0.04-0.15 \mathrm{~Hz}$ and $0.15-0.4 \mathrm{~Hz}$ respectively. LF and HF were quantified in normalized units in order to minimize the impact of difference in total power.

\section{PREDICTION OF COGNITIVE STRESS}

Using the physiological parameters measured with the camera as features, we build and test a classifier for predicting whether an individual is under cognitive stress or at rest. 


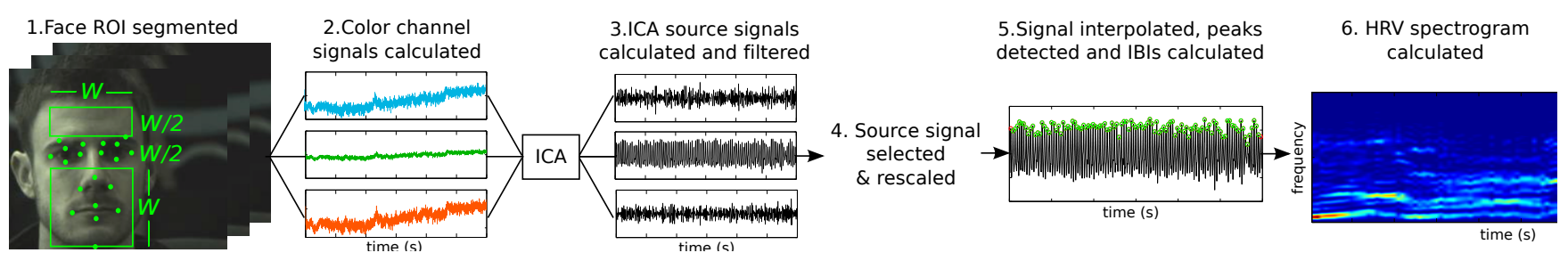

Fig. 3. Overview of the automated method used to recover the HRV spectrogram from videos of a human face. 1) Facial landmarks are detected using [15] and the face region of interest (ROI) segmented (excluding the region around the eyes), 2) spatial average of each color channel in the ROI over time calculated, 3) source signals, calculated via Independent Component Analysis (ICA), are filtered, 4) the channel with the estimated strongest BVP signal selected and inverted if necessary, 5) BVP signal interpolated to $256 \mathrm{~Hz}$, peaks detected, and IBIs calculated, 6) spectrogram calculated with a moving window (length 60 s, step size $1 \mathrm{~s}$ ).

We test a Naive Bayes model and a support vector machine (SVM) with linear kernel for the classification. SVMs have been shown to perform well on many classification problems. The input features were the mean heart rate, mean breathing rate, normalized HRV LF power, normalized HRV HF power and HRV LF/HF power ratio for each session. The cost, $C$, parameter was set to 0.1 . We performed a person-independent testing by withholding the data for one participant in the test set and using all the remaining data for training. We repeated this 10 times, once for each participant.

\section{RESULTS}

\section{A. Validation of Remote Physiological Measurements}

We compare the physiological parameters calculated using the camera measurements to those calculated from the contact sensor measurements. The correlations between the contact and remote methods were: $\mathrm{HR}=1.0, \mathrm{BR}=0.93$, $\mathrm{HRV} \mathrm{LF}=0.93, \mathrm{HRV} \mathrm{HF}=0.93$ and $\mathrm{HRV} \mathrm{LF} / \mathrm{HF}$ ratio $=0.93, p<0.01$ for all. The root mean squared errors for the HR, RR and HRV LF/HF were 0.106, 1.53 and 0.145 respectively. High agreement was observed and shows that the camera can accurately capture the BVP signal.

\section{B. Impact of Cognitive Stress on Physiology}

We compare the remotely measured physiological parameters for each of the participants. Figure 4 (a-d) shows the values of $\mathrm{HR}, \mathrm{BR}, \mathrm{HRV} \mathrm{LF}$ and $\mathrm{HRV} \mathrm{LF} / \mathrm{HF}$ ratio for each participant $($ blue $=$ rest, red $=$ session). Figure $4(\mathrm{e}-$ g) shows the mean HR, BR and HRV LF/HF ratio across all participants with error bars showing one standard deviation either side of the mean. Figure 5 shows examples of remotely measured HRV spectrograms from three participants at rest (top row) and under cognitive stress (bottom row). Using a sixty second window over a two minute video gave sixty second spectrograms. The higher LF/HF ratio under cognitive stress is evident. For the significance measures twosample Kolmogorov-Smirnov tests were used.

\section{Prediction of Stress from Physiology}

The prediction accuracy of the model for classifying rest or cognitive stress using a linear SVM was $85 \%$, this is a two-class case with balanced class sizes and therefore a naive prediction would be 50\%. Table I shows the accuracy using the HR, BR and HRV features alone and in combination.
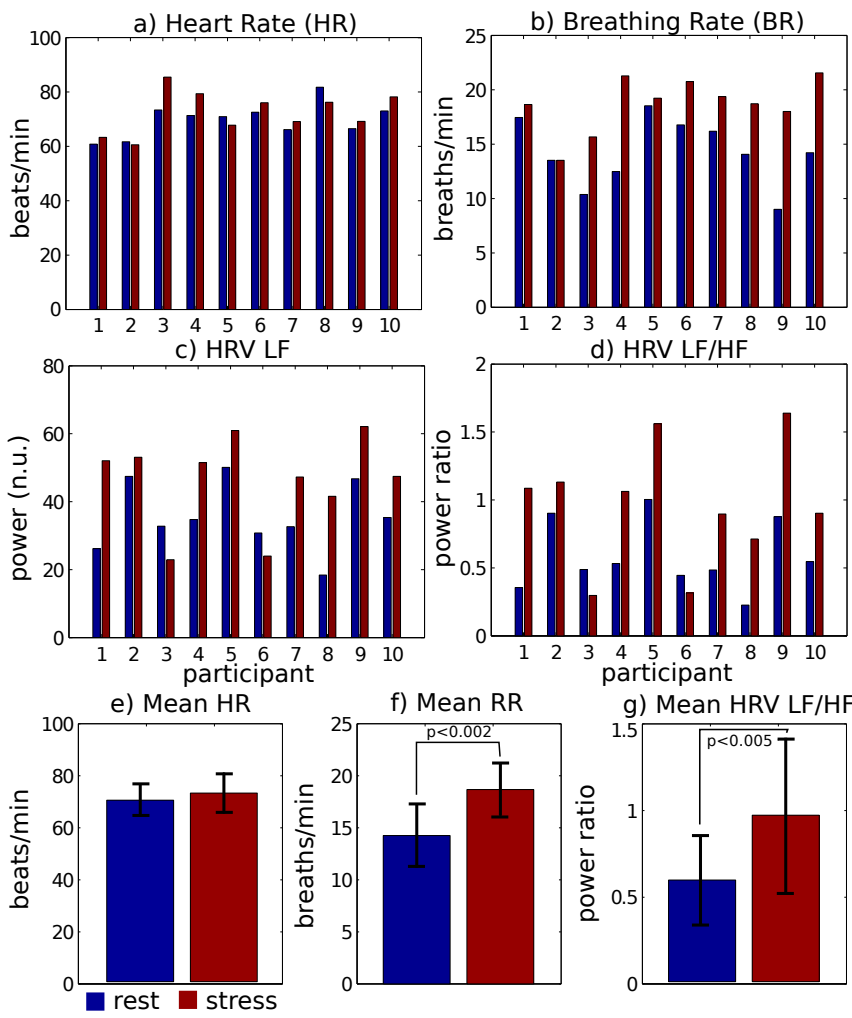

Fig. 4. Comparison of remotely measured physiological parameters for each participant during rest and stress. a) Heart rate, b) breathing rate, c) HRV LF, d) HRV LF/HF ratio. Mean parameters are shown for: e) heart rate, f) breathing rate and g) HRV LF/HF. Error bars show one standard deviation either side of the mean. Breathing rate and LF/HF ratio are the most discriminative features, heart rate was the least discriminative.

\section{DISCUSSION}

The physiological measurements made using the camera were highly correlated with the contact sensor measurements and are sensitive enough to capture differences between the responses of participants at rest and under cognitive stress. For $90 \%$ of the participants the breathing rate was higher during the cognitive stress session than at rest. The mean breathing rate across all participants during the stress sessions was significantly higher than during the rest sessions $(p<0.002)$. For $80 \%$ of the participants the HRV LF components were higher during the cognitive stress session. The mean HRV LF/HF ratio across all participants during the stress sessions was also significantly higher than during the rest sessions $(p<0.005)$, this supports the results in [4]. 
TABLE I

CLASSIFICATION ACCURACY FOR PREDICTION OF REST VS. STRESS USING DIFFERENT COMBINATIONS OF FEATURES.

\begin{tabular}{cccccc}
\hline Accuracy (\%) & Random & HR & BR & HRV & All \\
\hline \hline Naive Bayes & 50 & 65 & 75 & 70 & 80 \\
SVM & 50 & 60 & 75 & 70 & $\mathbf{8 5}$
\end{tabular}

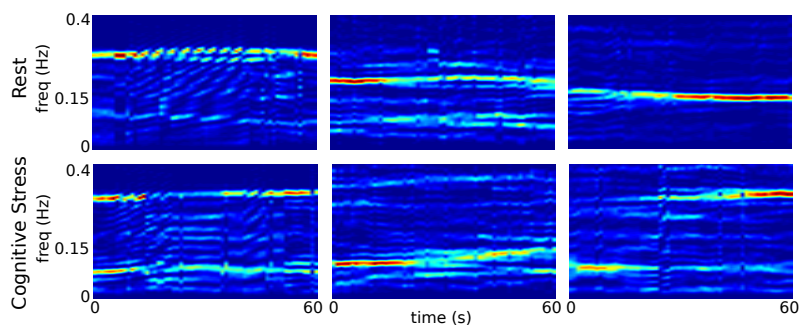

Fig. 5. Remotely measured HRV spectrograms from three participants at rest (top row) and under cognitive stress (bottom row). LF PSD components are measured from $0.04-0.15 \mathrm{~Hz}$, HF PSD components are measured from $0.15-0.4 \mathrm{~Hz}$. The higher $\mathrm{LF} / \mathrm{HF}$ ratio under cognitive stress is evident.

However, unlike [4] heart rate alone was not a strong predictor of cognitive stress, there was no significant difference between the mean heart rates in the rest and stress condition. The remotely measured spectrograms (Figure 5) show that the dominant frequency for all participants shifted between the rest and stress conditions. In addition the dominant frequency was not stationary during each session, showing why HRV spectrograms can provide useful information about the dynamics of a response.

The SVM classifier prediction accuracy was very good even with a challenging person-independent training scheme, $85 \%$ (17 out of 20) of sessions were correctly labeled as rest or cognitive stress. HRV components and breathing rate were the strongest predictors of cognitive stress. The responses of participants two and three under stress were misclassified as rest, they both showed relatively slow BRs and participant three had the lowest HRV LF/HF ratio under cognitive stress. The response of participant five at rest was misclassified as under stress, the highest BR was measured in that condition. Perhaps this was because the participant found the experiment, and having to wear contact sensors, stressful. In this case the rest condition may not have represented a truly relaxed state. All other sessions were correctly classified.

\section{CONCLUSIONS AND FUTURE WORK}

We present remote measurement of physiological parameters using a digital camera (at a distance of $3 \mathrm{~m}$ ). We captured the physiological responses of participants at rest and under cognitive stress. The remote measurement of heart rate, breathing rate and heart rate variability closely agreed with contact measurements. Significant differences were observed in the remotely measured BRs and HRV components between the states of rest and cognitive stress. Heart rate was not significantly different between the rest and stress conditions. We designed a person-independent classifier for predicting whether a participant was under cognitive stress or at rest from the remotely measured physiology. The accuracy of the model was $85 \%$ much higher than a naive prediction. The BR and HRV features were the most discriminative.
This work suggests it is possible to remotely measure an individual's cognitive load using a digital camera, a finding that could be very useful for workplace stress measurement or in learning contexts. Different types of stressors (e.g. physical, emotional, cognitive) are likely to result in different types of responses, future work will investigate the impact of other stressors on physiological responses. Although participants were free to move during the experiments they remained facing the camera at all times. The effects of greater rigid head motions and facial expressions will be studied in future work.

\section{REFERENCES}

[1] S. Akselrod, D. Gordon, F. A. Ubel, D. C. Shannon, A. Berger, and R. J. Cohen, "Power spectrum analysis of heart rate fluctuation: a quantitative probe of beat-to-beat cardiovascular control," science, vol. 213, no. 4504, pp. 220-222, 1981.

[2] A. Moriguchi, A. Otsuka, K. Kohara, H. Mikami, K. Katahira, T. Tsunetoshi, K. Higashimori, M. Ohishi, Y. Yo, and T. Ogihara, "Spectral change in heart rate variability in response to mental arithmetic before and after the beta-adrenoceptor blocker, carteolol," Clinical Autonomic Research, vol. 2, no. 4, pp. 267-270, 1992.

[3] N. Hjortskov, D. Rissen, A. K. Blangsted, N. Fallentin, U. Lundberg, and K. Søgaard, "The effect of mental stress on heart rate variability and blood pressure during computer work," European journal of applied physiology, vol. 92, no. 1-2, pp. 84-89, 2004.

[4] Z. B. Moses, L. J. Luecken, and J. C. Eason, "Measuring taskrelated changes in heart rate variability," in Engineering in Medicine and Biology Society, 2007. EMBS 2007. 29th Annual International Conference of the IEEE. IEEE, 2007, pp. 644-647.

[5] E. Kristal-Boneh, M. Raifel, P. Froom, and J. Ribak, "Heart rate variability in health and disease," Scandinavian journal of work, environment \& health, pp. 85-95, 1995.

[6] R. El Kaliouby and P. Robinson, "Real-time inference of complex mental states from facial expressions and head gestures," in Real-time vision for human-computer interaction. Springer, 2005, pp. 181-200.

[7] D. Shastri, B. Bass, I. Pavlidis, M. Papadakis, and P. Tsiamyrtzis, "Perinasal imaging of physiological stress and its affective potential," IEEE Transactions on Affective Computing, vol. 3, no. 3, pp. 366-378, 2012.

[8] G. Lu, F. Yang, J. Taylor, and J. Stein, "A comparison of photoplethysmography and ecg recording to analyse heart rate variability in healthy subjects," Journal of Medical Engineering \& Technology, vol. 33, no. 8, pp. 634-641, 2009.

[9] J. Allen, "Photoplethysmography and its application in clinical physiological measurement," Physiological measurement, vol. 28, no. 3, p. R1, 2007.

[10] W. Verkruysse, L. O. Svaasand, and J. S. Nelson, "Remote plethysmographic imaging using ambient light," Optics express, vol. 16, no. 26, pp. 21434-21 445, 2008.

[11] M.-Z. Poh, D. J. McDuff, and R. W. Picard, "Non-contact, automated cardiac pulse measurements using video imaging and blind source separation," Optics Express, vol. 18, no. 10, pp. 10 762-10 774, 2010.

[12] — "Advancements in noncontact, multiparameter physiological measurements using a webcam," Biomedical Engineering, IEEE Transactions on, vol. 58, no. 1, pp. 7-11, 2011.

[13] D. McDuff, S. Gontarek, and R. Picard, "Improvements in remote cardio-pulmonary measurement using a five band digital camera," Biomedical Engineering, IEEE Transactions on, 2014.

[14] G. Balakrishnan, F. Durand, and J. Guttag, "Detecting pulse from head motions in video," in Computer Vision and Pattern Recognition (CVPR), 2013 IEEE Conference on. IEEE, 2013, pp. 3430-3437.

[15] B. Martinez, M. F. Valstar, X. Binefa, and M. Pantic, "Local evidence aggregation for regression-based facial point detection," IEEE Transactions on Pattern Analysis and Machine Intelligence, vol. 35, no. 5, pp. 1149-1163, 2013.

[16] T. E. Brown, L. A. Beightol, J. Koh, and D. L. Eckberg, "Important influence of respiration on human rr interval power spectra is largely ignored," Journal of Applied Physiology, vol. 75, no. 5, pp. 23102317, 1993. 